\title{
Guerre totale et/ou force minimale ? Histoire et paradoxes des « cœurs et des esprits »
}

\section{Christian Olsson}

\section{OpenEdition}

\section{Journals}

Édition électronique

URL : http://journals.openedition.org/conflits/3102

DOI : $10.4000 /$ conflits.3102

ISSN : $1777-5345$

Éditeur :

CCLS - Centre d'études sur les conflits lilberté et sécurité, L'Harmattan

Édition imprimée

Date de publication : 15 novembre 2007

Pagination : $35-62$

ISBN : 978-2-296-04582-8

ISSN : 1157-996X

Référence électronique

Christian Olsson, «Guerre totale et/ou force minimale? Histoire et paradoxes des « cœurs et des esprits » », Cultures \& Conflits [En ligne], 67 | automne 2007, mis en ligne le 04 janvier 2010, consulté le 30 mars 2021. URL : http://journals.openedition.org/conflits/3102 ; DOI : https://doi.org/10.4000/ conflits.3102 


\section{Guerre totale et/ou force minimale? Histoire et paradoxes des « cœurs et des esprits» 1}

\section{Christian OLSSON}

Christian Olsson achève actuellement sa thèse de science politique à l'IEP de Paris sur la "légitimation locale de la force militaire internationale". Il est par ailleurs chercheur junior dans le cadre du programme de recherche Challenge (PCRD 6), chercheur associé au Centre d'études sur les conflits et membre du réseau européen de recherche CASE (Critical Approaches to Security in Europe).

«Chaque fois que les incidents de guerre obligent l'un de nos officiers à agir contre un village [...], il ne doit pas perdre de vue que son premier soin, la soumission des habitants obtenue, sera de reconstruire le village, d'y créer un marché, d'y établir une école. C'est de l'action de la politique et de la force que doit résulter la pacification du pays et l'organisation à lui donner plus tard ».

Général Gallieni, instructions fondamentales du 22 mai 1898 à Madagascar

« [...] c'est dans le village que l'on sécurise par la force qu'il faudra demain rétablir des conditions de vie normales, recréer le marché et envoyer les enfants aux écoles. [...]. La diplomatie de terrain et l'action militaire se conjuguent et sont deux des visages du soldat en campagne ».

CDEF, «Gagner la bataille, conduire à la paix : les forces terrestres dans les conflits aujourd'hui et demain », FT01, janvier 2007, p. 21

1. Cet article est notamment le fruit d'une recherche de terrain effectuée en Afghanistan en septembre 2007, qui a permis de conduire un certain nombre d'entretiens avec des troupes de la Force internationale d'assistance à la sécurité, FIAS (notamment du QG et du commandement régional est) et d'Operation Enduring Freedom, OEF (Task Force Phoenix, Camp Julien), ainsi que d'un grand nombre d'entretiens réalisés entre autres auprès de militaires français, britanniques et américains en dehors de ce théâtre. 

'idée que les militaires en « opération extérieure »(Opex) en Afghanistan, en Irak, mais aussi en Côté d'Ivoire, au Congo et ailleurs, ont notamment vocation à « conquérir », à " gagner » ou à « vaincre » dans la bataille pour « les cœurs et les esprits des populations locales », dans le cadre d'opérations de «stabilisation », de « contre-insurrection » ou " de paix » semble désormais un lieu commun. La référence aux « cœurs et aux esprits » (ou en anglais : « hearts and minds ») devient ainsi un cliché dont on use et abuse volontiers ad nauseam sans que celui-ci ne soit jamais interrogé, analysé ou inscrit dans une histoire dépassant les seules préoccupations du temps présent. Dès lors, cet article souhaite retracer la genèse - au travers d'exemples tirés des doctrines et pratiques militaires américaines, françaises et britanniques - de ce leitmotiv, pour mieux en jauger les enjeux contemporains sous-jacents. Cet énoncé discursif fournira donc ici un des biais par lequel nous tenterons de reconstruire les continuités historiques en dehors desquelles il est difficile de dégager les lignes de force, et donc les enjeux, qui traversent les pratiques militaires contemporaines.

L’idée de la « conquête des cour et des esprits » est aujourd'hui tellement omniprésente que certains en transposent les recettes sur les enjeux de sécurité intérieure par le biais de l'argument de la menace d'une « insurrection globale 2 ». Cependant, pour ce qui est du temps présent, nous ne considèrerons ici que les opérations militaires extérieures, et plus particulièrement celles menées en Irak ${ }^{3}$ et en Afghanistan ${ }^{4}$. En effet, les doctrines anglo-saxonnes de la contre-insurrection qui y sont aujourd'hui ré-actualisées - ainsi que leurs " prédécesseurs » historiques anglo-américains (small wars, imperial policing, unconventional warfare, military nation building, foreign internal defense, internal defense and development, low intensity conflicts, etc.) mais aussi, dans une moindre mesure, français (doctrine de la « guerre révolutionnaire » notamment) - sont à la fois à l'origine et intrinsèquement liés à l'énoncé « des cœurs et des esprits ».

Dans une certaine mesure, les analyses qui suivent concernent également directement ou indirectement d'autres types d'approches, notamment celles contemporaines en termes de "stabilisation », ou même parfois celles en termes $d$ ' « opération de paix ». Bien évidemment ces approches ne sont pas identiques. Cependant, les frontières conceptuelles et doctrinales sont parfois plus perméables qu'il n'y paraît. Alors qu'il est facile d'établir des taxinomies des différentes types d'approches et doctrines en Opex selon des critères divers, nous entendons ici insister - et il s'agit d'un choix méthodologique de notre part ${ }^{5}$ - sur ce qu'el-

2. Kilcullen D., "Countering global insurgency: a strategy for the war on terrorism”, Journal of Strategic Studies, vol. 28, n4, août 2005, pp. 519-617; Mackinlay J., Globalization and Insurgency, Adelphi Paper 352, Londres, International Institute for Strategic Studies (IISS), novembre 2002.

3. Coalition multinationale Irak.

4. Operation Enduring Freedom (OEF) et Force internationale d'assistance à la sécurité (FIAS).

5 . Le risque de toute démarche historique est d'avaliser inconsciemment les catégories préconstruites du temps présent et de les projeter dans le passé. Comme le souligne Gérard Noiriel, 
les ont en commun dans la mesure où notre objet spécifique, celui des discours et pratiques de la «conquête des cœurs et des esprits », tend à s'étendre à nombre d'entre elles. Par ailleurs, en dépit des différences parfois réelles, elles puisent souvent dans une histoire commune et, qui plus est, les distinctions sont parfois floues, voire inexistantes, pour les professionnels même qui définissent ces approches et doctrines ${ }^{6}$.

Cet article, librement inspiré par la généalogie foucaldienne des technologies de pouvoir, sera structuré en trois parties. Tout d'abord, nous dresserons les contours de notre objet d'analyse en insistant notamment sur le rôle joué par les savoir-faire militaires coloniaux dans la définition de certaines doctrines contemporaines. Nous essaierons ensuite de montrer plus spécifiquement en quoi et comment ces savoir-faire sont aujourd'hui réactualisés dans les Opex en Afghanistan et en Irak. Enfin, nous tenterons de montrer comment l'analyse historique peut permettre de décrypter et d'expliciter certains des enjeux sousjacents aux doctrines contre-insurrectionnelles contemporaines.

\section{"Voir le passé dans le présent » : contre-insurrection et probléma- tique coloniale}

Au début des années 1990, on a beaucoup parlé des «nouvelles Opex ». Pourtant, les évocations des similarités structurelles entre les missions « interventionnistes » contemporaines et les engagements passés, notamment dans les contextes coloniaux, ne manquent pas ${ }^{7}$. Mais à la différence de ce qui est généralement fait, nous essaierons ici d'analyser les continuités coloniales avec une certaine systématicité - mais sans exhaustivité aucune - tout en essayant d'en dégager les enjeux pertinents à la compréhension du temps présent. De surcroît, il s'agit ici d'actualiser la problématique à l'aune des transformations récentes des pratiques militaires en Afghanistan et en Irak au travers desquelles elle a indéniablement gagné en saillance. Nous essaierons ainsi de montrer que certains des principes sous-jacents aux tactiques, techniques et procédures («technics, tactics and procedures ", TTP) militaires mises en œuvre n'y ont pas émergé ex nibilo malgré les récits fréquents sur l'improvisation dans le feu de l'action.

toute récit socio-historique voulant éviter les travers de «l'histoire historicisante » se doit alors d'éviter de naturaliser les découpages conceptuels opérés par le langage contemporain. Il faut au contraire construire et définir sa problématique intellectuelle et son objet avant d'identifier les ressources documentaires pertinentes. Voir : Noiriel G., Introduction à la socio-histoire, Paris, La Découverte, 2006.

6. Ce fait a été bien perçu par Frank Kitson, le héraut britannique de la contre-insurrection dans les années 1960 et 1970, qui utilisait volontiers les expressions « counter-insurgency », «stability operations ", «low intensity warfare » et "peacekeeping » comme équivalents sémantiques. Voir : brigadier Kitson F., Low Intensity Operations: subversion insurgency and peacekeeping, London, Faber and Faber, 1971.

7. On se reportera notamment à Mockaitis T.R., "From counterinsurgency to peace enforcement: new names for old games?", in Schmidl E.A., Peace Operations between peace and war, London, Frank Cass, 2000, pp. 21-36 ; Paulmier T., «Les opérations de maintien de la paix : l'incertitude sur les nouvelles missions de l'armée française », Les Champs de Mars, n³, 1998, pp. 89-106. 
En effet, le « temps présent » n’est jamais un ordre émergeant spontanément du néant, à la fois autonome, statique et auto-constitutif. Faire abstraction du passé, c'est naturaliser et réifier le présent et ainsi empêcher de voir qu'il est fait de processus et de sédimentations multiples en dehors desquels tout questionnement intellectuel deviendrait vain. Il faut alors voir que les pratiques militaires s'inscrivent notamment dans des histoires qui dépassent la seule temporalité de l'action militaire. Mais encore une telle entreprise doit-elle savoir éviter l'écueil des fausses continuités rétrospectives. Ainsi, elle ne saurait consister en une relecture du passé au travers du prisme du présent ${ }^{8}$, une simple projection du présent dans le passé qui reviendrait à écrire une histoire linéaire (téléologique) ou cyclique (isomorphisme du présent par rapport à d'autres périodes) d'avènement nécessaire du présent. Il faut, en d'autres termes, s'efforcer avec Paul Veyne de voir que les pratiques et les discours des acteurs sociaux n'ont pas visé à atteindre l'objectif que, pourtant, ils peuvent avoir historiquement contribué à actualiser - de par leurs interactions - à partir des conditions de possibilité de leur temps présent ${ }^{9}$. L'analyse du passé permet alors d'identifier les lignes d'hétérogénéité malgré les continuités historiques, les continuités historiques malgré les changements et ainsi, au final, de «voir le passé dans le présent 10 ».

\section{Les « autres guerres » et l'émergence des «populations » dans le discours militaire}

La guerre, celle que les Etats modernes ont connue, celle dont la grande majorité des livres de stratégie militaire traitent, cette guerre «industrielle » n'existe plus. Cela n'est pas la conclusion d'un pacifiste mais d'un général britannique, le général Rupert Smith ${ }^{11}$. Il montre que les conflits contemporains impliquant des militaires " occidentaux », qu'il appelle les "guerres parmi la population» ("war amongst the people»), sont radicalement différents des guerres interétatiques classiques. En effet, l'enjeu stratégique y est l'adhésion - relevant souvent, dans ce type de discours, à le fois des intérêts, de la légitimation et de la coercition - de la « population locale » au mandat politique des militaires et non l'épreuve décisive de la force sur le champ de bataille. Il en découle que les militaires doivent savoir conformer leurs pratiques - notamment au niveau «tactique »- à ce nouvel impératif politico-stratégique ou, du moins, tenter d'infléchir les allégeances, les représentations et les intérêts de cette population dans un sens favorable aux militaires et à leur mandat. Dans ce dernier cas, on dira volontiers qu'il s'agit de «conquérir les cœurs et les esprits des populations locales ». Concrètement, cette conquête peut passer par des mesures «positives» (valorisation des actions des forces armées, notamment par des opérations psychologiques), « négatives » (stigmatisation

8. Pour une critique de cet écueil voir: Hobden S., Hobson J.M., Historical Sociology of International Relations, Cambridge, Cambridge University Press, 2002.

9. Veyne P., «Foucault révolutionne l'histoire », Comment on écrit l'histoire, Paris, Seuil, 1978, pp. 385-429.

10. Voir Noiriel G., op. cit.

11. Smith R., The Utility of Force, The Art of War in the Modern World, Londres, Alan Lane, 2005. 
de l'adversaire) et structurelles («réformisme» politique, économique et social jouant sur les ressorts perçus du mécontentement) dans ce qui est lu comme une guerre de légitimité dont l'enjeu principal est « la population », ses caractéristiques propres, ses craintes, ses convictions, ses perceptions et la manière d'en tirer profit et/ou de les infléchir. Il n'est alors pas étonnant que ce soient les lieux de concentration des populations, les villes, qui retiennent l'essentiel de l'intérêt et qui constituent, consciemment ou non, le modèle sous-jacent à ces approches ${ }^{12}$.

Mais si ces réflexions stratégiques sur ces " guerres non conventionnelles », ces «autres guerres» - notamment dans le cadre des Opex - paraissent aujourd'hui centrales dans la redéfinition du rôle des militaires, elles n'en sont pas pour autant nouvelles. Pendant la Guerre froide, ces guerres ont souvent été pensées à l'aune de la confrontation bipolaire. Elles étaient alors abordées en tant que "conflits périphériques » découlant d'une stratégie de "l'approche indirecte »- selon l'expression de Liddell Hart ${ }^{13}$ - de la part de l'URSS pour subvertir l'équilibre des puissances. Le savoir stratégique dominant, notamment pendant la Guerre froide, a ainsi souvent empêché d'analyser ces conflits dans leur singularité pour réduire leurs acteurs - lorsque défavorables au bloc occidental - à une « cinquième colonne » au service du bloc communiste qu'il fallait notamment savoir «neutraliser » dans le cadre d'une guerre conventionnelle généralisée ${ }^{14}$. Mais cela n'a pas empêché un savoir spécifique sur ces conflits de se développer à l'ombre du paradigme dominant de la dissuasion nucléaire. Or, ce savoir stratégique périphérique, que ce soit sous la forme de la « contre-insurrection », de la « guerre révolutionnaire », « anti-subversive », de «faible intensité » ou de « contre-guérilla » dans les espaces coloniaux et postcoloniaux - savoirs eux-mêmes inspirés de la «pacification coloniale » à la française ou de la «police impériale » britannique, toutes deux bien antérieures à la Guerre froide -, nous apprend deux choses importantes pour notre propos.

Premièrement ces savoirs, et les savoir-faire qui en découlent, structurent fortement les discours doctrinaux et stratégiques contemporains sur ce que Rupert Smith appelle la « guerre parmi la population », expression désormais reprise dans la doctrine de l'armée de terre française ${ }^{15}$. Ainsi, on peut noter à titre d'exemple que l'expression "guerre dans la foule ", lancée en 1956 par le colonel Jean Némo pour décrire le type d'opérations dans lesquelles l'armée française était engagée en Indochine et en Algérie 16 a précédé de 50 ans celle de "guerre

12. Ce qui ne manque d'ailleurs pas de poser des problèmes à la contre-insurrection rurale, notamment en Afghanistan.

13. Sir Liddell Hart B., Stratégie, Paris, Albin Michel, 2001.

14. Général Beaufre A., Introduction à la stratégie, $3^{\mathrm{e}}$ éd., Paris, Armand Colin, 1965.

15 . «Doctrine d'emploi des forces terrestres en stabilisation », Centre de doctrine d'emploi des forces, 23 novembre 2006 ; « Gagner la bataille, conduire à la paix, les forces terrestres dans les conflits aujourd'hui et demain », FT01, Centre de doctrine d'emploi de forces, Paris, janvier 2007.

16. Colonel Nemo J., « La guerre dans la foule », Revue de Défense nationale, juin 1956, pp. 721-734. 
parmi la population » pourtant présentée comme une innovation conceptuelle majeure. Par ailleurs, les références aux auteurs britanniques de la contre-insurrection coloniale et post-coloniale (Thompson et Kitson ${ }^{17}$ ) ou français de la « guerre révolutionnaire » 18 (Trinquier, Galula, Lacheroy, Beaufre, etc.) et de la pacification coloniale (Lyautey et Gallieni) sont pléthore dans les textes doctrinaux contemporains traitant de l'engagement des militaires dans les « nouveaux conflits de projection ».

Deuxièmement, il ne s'agit pas d'un savoir stratégique et doctrinal homogène et consolidé, mais d'un ensemble disparate d'éléments hétérogènes et d'une infinité de nuances qu'il faut à chaque fois replacer dans leur contexte d'origine. Cependant, une de leurs caractéristiques communes est de souvent placer les "populations », les «masses» ou encore les «foules» 19 - et non pas le front linéaire séparant l'ami de l'ennemi comme dans les « guerres clausewitziennes »au centre des considérations. Il s'agit des populations à l'intérieur de l'Etat (ou de la métropole) en guerre « en externe »- on parlera alors souvent de « front intérieur »- mais aussi et surtout des populations «locales» sur le territoire sur lequel les militaires sont engagés. Dans ce cas, on a souvent parlé de « guerre en surface » par opposition à celle sur un front linéaire. L'enjeu est en effet notamment d'identifier, de localiser, de fixer, d'isoler et de «neutraliser » l'ennemi se cachant au sein de la population. Sur un plan plus général, cela suppose de connaître, de contrôler et, si possible, d'obtenir l'allégeance, la loyauté, le consentement, l'approbation ou à défaut, l'acquiescement, l'assujettissement ou la soumission, de ces populations. En ce sens, ces engagements se distinguent des guerres classiques ou «trinitaires» selon le terme de van Creveld, fondées sur la distinction claire entre gouvernement, forces armées et populations. Ils voient en effet s'opérer une interpénétration des facteurs politiques, des facteurs militaires et des enjeux liés à la « gestion des populations » 20 . On considèrera alors souvent dans le langage militaire que la population est le «centre de gravité »- expression de Clausewitz désignant originellement le lieu de concentration de la puissance militaire des armées ennemies - de ces conflits. Il s'agira alors de « conquérir les cœurs et les esprits » de ces « populations » ou de cette " population », afin que l'ennemi « irrégulier » ne puisse s'y infiltrer, s'y ravitailler et s'y multiplier.

En suivant les analyses de Foucault, on peut constater que dans ces types de discours la «population » apparaît essentiellement - comme dans les dispositifs de sécurité contemporains - en tant que sujet (le « public ») mais aussi

17. Voir notamment: Thompson R., Defeating Communist Insurgency: Experiences from Malaya and Vietnam, New York, Frederick a. Praeger, 1966.

18. Sur ces auteurs, voir notamment: Périès G., La doctrine française de la "guerre révolutionnaire": Indochine, Algérie, Argentine, Rwanda, trajets d'une hypothèse, mémoire de HDR, 2006 ; Périès G., Servenay D., Une guerre Noire, enquête sur les origines du génocide rwandais (1959-1994), Paris, La Découverte, 2007.

19. Sur la notion de «foule » dans le vocable militaire voir : Potier E., « Imaginaire du contrôle des foules dans l'armée de terre française », Cultures $\mathcal{E}$ Conflits, n56, 2004, pp.35-49.

20. Van Creveld M., The Transformation of War, New York, Free Press, 1991. 
en tant qu'objet technico-politique de gouvernement ${ }^{21}$. Il ne s'agit en effet pas de la somme des individus vivant dans un espace, dans un « milieu ", mais de l'ensemble de ces individus auquel on a soustrait « l'ennemi », celui sur lequel le pouvoir «normalisateur» (puisqu'il s'agit, dans le vocabulaire militaire français, de tendre vers la «normalisation ») de l'entreprise interventionniste n'a pas prise. Ce type de discours doctrinal est en effet inséparable de la distinction binaire entre population et ennemi (terroriste, insurgé ou guérilla) sans jamais que ce dernier soit considéré comme une sous-catégorie de la première. Il ne saurait en effet être question de faire la guerre à (une partie de) la «population », mais seulement de faire la guerre "parmi la population ». Il faut séparer l'ennemi de la «population », physiquement et conceptuellement, comme s'il s'agissait de deux entités incommensurables. Il découle de cette distinction artificielle que lorsque la «population » apporte son soutien à l'« ennemi », ce ne peut être que parce que ce dernier l'instrumentalise en la réduisant, depuis un extérieur indéfini, à une variable technique servant un objectif qui échappe à cette même population. C'est peut être cette logique instrumentale prêtée à l'ennemi qui explique pourquoi, dans une certaine mesure, les doctrines contre-insurrectionnelles - dont les concepteurs, en tant que stratèges ou stratégistes, se revendiquent également d'une rationalité instrumentale considérant «l'autre » essentiellement comme variable technique 22 - se construisent par mimétisme par rapport à l'analyse faite de l'adversaire, et cela dès l'époque coloniale.

Mais encore faut-il s'accorder sur ce que l'on peut ici entendre par « colonial », terme dont les sens et connotations sont très variables ${ }^{23}$. Le terme est ici utilisé pour désigner une période - celle qui s'ouvre avec les grandes découvertes au $\mathrm{XVI}^{\mathrm{e}}$ siècle et se clôt théoriquement avec la décolonisation - mais aussi une pratique - la mise sous influence politique de territoires « allogènes ». Plus précisément, il s'agit ici de la colonisation s'accompagnant de domination politique de territoires et de sujétion de leurs populations. Aussi, nous y inclurons non seulement les colonies au sens propre du terme, mais aussi les protectorats, les dominions et les territoires rattachés tels que l'Algérie pour la France ou les Philippines à la suite de la guerre de 1899-1902 pour les Etats-Unis. Il faut également souligner que la période de décolonisation a prolongé certaines pratiques de « gouvernance à distance » d'Etats post-coloniaux, notamment par adaptation de la technique coloniale britannique du «indirect rule ». On parlera à ce

21. Foucault M., Sécurité, territoire, Population, Cours au Collège de France, 1977-1978, Paris, Seuil/Gallimard, 2004.

22. Pour une approche critique de la tradition stratégique, voir : Wyn Jones R., Security, Strategy, and Critical Theory, Colorado, Lynne Rienner, 1999.

23. Ce qui a notamment permis aux représentants américains de toujours nier officiellement la dimension coloniale de certains épisodes de l'histoire américaine. Sur cette négation, malgré les dimensions spécifiquement coloniales de la contre-insurrection américaine, notamment au Vietnam, voir : Latham M.E., Modernization as Ideology: American Social Science and "Nation Building” in the Kennedy Era, Chapel Hill, University of North Carolina Press, 2000. 
propos de «néo-colonialisme ». Il nous faut alors aborder les genèses historiques du savoir-faire militaire colonial pour mettre en évidence un certain nombre de traits distinctifs et de caractéristiques pertinents - comme nous le verrons par la suite - à la compréhension du temps présent.

Genèses des savoir-faire militaires de "sécurité intérieure » dans les contextes coloniaux

Décrire dans l'espace d'un article l'ensemble des pratiques développées pour lutter contre les formes de violence et d'insoumissions populaires protéiformes dans le contexte des expériences coloniales et néo-coloniales depuis le XIXe siècle, relève de la gageure. Ces pratiques sont très diversifiées selon les puissances coloniales, mais aussi selon la zone régionale considérée. Elles vont cependant constituer un fond commun de savoir-faire colonial de sujétion des populations puis de maintien de l'ordre, même si celui-ci sera encore largement mis en œuvre de manière ad hoc au XIXe siècle. Ce fond commun évoluera considérablement depuis la pacification coloniale française, les «small wars » puis la « police impériale » britanniques, jusqu'aux guerres de décolonisation. En effet, au fur et à mesure que le seul recours à la force létale fondé sur le principe de la "punition collective » montre ses limites en accroissant l'insoumission et les résistances populaires, des pratiques de gestion des populations et de légitimation de la force (éducation, assistance médicale, développement rural) sont développées, à l'instar des Bureaux arabes en Algérie puis des Affaires indigènes au Maroc, suivant ainsi une logique d'économie (toute relative dans le cas des contextes coloniaux) de la coercition que Foucault a également mise en évidence à propos de l'évolution des institutions pénales en Europe ${ }^{24}$. Dans les années 1950 et 1960, ce savoir-faire sera théorisé, formalisé et mis en cohérence sous différentes formes.

Ces formalisations, que ce soit au travers de la contre-insurrection américaine, de la contre-insurrection britannique ou de la guerre révolutionnaire française, sont pour partie (mais pour partie seulement) une mise en système et une synthèse renouvelée des enseignements disparates tirés des expériences précédentes des «troubles» dans les colonies ${ }^{25}$. Les échanges de savoirs et de savoirfaire entre puissances coloniales deviendront également de plus en plus fréquents à cette période. On peut ainsi isoler les éléments communs de ces doctrines. Cela est d'autant plus vrai qu'à partir des années 1950, la référence à la pensée de Mao Zedong devient omniprésente au fur et à mesure que l'objectif de maintenir l'influence sur les colonies se double du souci de contrer l'avancée du communisme. Les perceptions militaires des « guérillas locales » sont ainsi homogénéisées derrière la figure unificatrice de l'insurrection et de la révolution communiste et, dès lors, ces doctrines ont notamment pour point commun d'affirmer, du moins formellement, la nature « politique » ou idéologique des « troubles». 
Il ne saurait ici être question de présenter les spécificités individuelles de chacune de ces doctrines ou approches. Pour synthétiser et mieux comprendre ces formalisations doctrinales - au risque de simplifier à outrance - on peut distinguer deux principes généraux d'une part et quatre catégories de pratiques d'autre part. Plus que des distinctions, il s'agit de continuum. Ces derniers sont faits d'éléments qui, loin d'être incompatibles, se combinent de différentes manières et ont plus ou moins d'importance selon les approches adoptées. Concernant les principes généraux, il s'agit premièrement de «neutraliser » les insurgés ou les guérillas après les avoir identifiés, localisés et fixés. Il s'agit notamment de ce qui est parfois appelé la « contre-guérilla». Cependant, au fur et à mesure que la nature politique de l'ennemi se fait plus évidente - sous l'influence du nationalisme et du communisme - et que la guérilla est de plus en plus perçue comme l'expression d'une poussée insurrectionnelle ou révolutionnaire, le deuxième principe général devient plus prégnant. Il s'agit de contrôler non pas tant le territoire que les représentations et les allégeances politiques des populations qui y vivent. Il s'agit de mobiliser, d'encadrer (y compris idéologiquement, notamment dans le cas de la "guerre révolutionnaire » française) et d'emporter l'adhésion des populations en les détournant de l'emprise politique de l'ennemi qui s'exercerait notamment par le «terrorisme ». En effet ce dernier, de par son impact psychologique, susciterait l'apathie chez les éléments les plus loyalistes, ferait basculer les opportunistes du côté de l'ennemi et galvaniserait les soutiens de ce dernier en provoquant les forces de sécurité coloniales à recourir à la force excessive et mal ciblée.

Pour contrer cette stratégie « insurrectionnelle », quatre catégories génériques d'action sont définies. Elles sont pour l'essentiel produites par un effet de mimétisme par rapport à l'analyse faite de l'adversaire ou de l'ennemi ${ }^{26}$. On les retrouve sous des formes et dans des proportions différentes dans toutes les campagnes de « pacification » dans le contexte de la décolonisation. Il s'agit premièrement d'éliminer l'ennemi, tout en évitant un usage « excessif » de la force voire en essayant de remplacer le principe militaire traditionnel en termes de " toute la force nécessaire 27 » ou de « force maximale » par une approche en termes de « force appropriée », de «force mesurée 28 » et, dans le cas de la tradition juridique britannique, par celui en termes de «force minimale 29 ». Dans la pratique cependant, contrairement à une vision commune qui tend à opposer les

26. Mc Clintock M., Instruments of Statecraft: U.S. Guerilla Warfare, Counterinsurgency, and Counterterrorism, 1940-1990, New York, Pantheon Books, 1992.

27. Brodeur J.-P., «Maintien et imposition de la paix en Somalie (1992-1995)", Cultures \& Conflits, n²9-30, 1998, pp.175-193.

28. Voir: The U.S. Army/Marine Corps Counterinsurgency Field Manual, University of Chicago Press, 2007, pp. 3-24.

29. Voir notamment: War Office (Code n ${ }^{\circ} 8439$ ), "Imperial policing and duties in aid of the civil power", Londres, Fosh and Cross, 13 juin 1949; Liddell Hart Centre for Military Archives; Catalog, 15/8/1-284. Il faut ici noter que la Grande-Bretagne lèguera cette tradition policière de « force minimale », tirée d'une interprétation dominante de la Common Law, aux opérations de maintien de la paix onusiennes. 
deux grandes puissances coloniales - la France et la Grande-Bretagne - d'une part et les Etats-Unis d'autre part, il semblerait que la France et les Etats-Unis auront tendance à maintenir des approches « conventionnelles » du recours à la force et cela, dans une certaine mesure, par opposition à la Grande-Bretagne 30 . Mais dans les deux cas, l'élimination de l'ennemi suppose d'abord de l'identifier et de le localiser. Ces tâches sont pour partie assurées au travers d'opérations de type policière menées par des petites unités militaires mobiles et autonomes coopérant étroitement avec des services de renseignement. Elles conduisent souvent à former des troupes supplétives et des polices paramilitaires « locales » qui auraient l'avantage de bien connaître l'environnement des opérations. Ainsi, il n'est pas rare que les « forces de sécurité » coloniales aient eu recours à d'anciens guérilléros reconvertis tels que les «bleus de chauffe » du capitaine Léger pendant la guerre d'Algérie.

Il s'agit deuxièmement de persuader les populations de la légitimité et de l'efficacité des troupes coloniales au travers d'actions psychologiques, de protection des communautés, mais aussi d'activités civiles de développement et d'assistance telle que celles menées par les équipes CORDS (Civil Operations and Rural Development Support) pendant la guerre du Vietnam ou les Sections administratives spécialisées (SAS) en Algérie. Ces pratiques sont au besoin complétées par des mesures de cooptation d'acteurs locaux influents et « ambitieux » que l'on pense susceptibles d'avoir des intérêts convergeant avec ceux des forces coloniales ${ }^{31}$. Il s'agit en effet, comme il a déjà été souligné, de conquérir «les cœurs et les esprits de la population », expression généralement attribuée à Sir Gerard Templer, responsable de la Malaisie pendant la campagne contreinsurrectionnelle du Malayan Emergency ( Urgence malaise » entre 19481960). Elle semble cependant déjà avoir été utilisée à propos de la pacification des tribus Pathan (Pachtoun) dans la zone de la Nortb Western Frontier du Raj britannique 32 et plus particulièrement $d u$ Waziristan et du Balouchistan dans les années 1920 33. Il s'agit alors notamment de répondre au "griefs légitimes » des populations locales, notamment économiques, sanitaires et éducationnels (par l'assistance économique et médicale), généralement en prenant grand soin de ne pas évoquer les « griefs politiques », considérés comme hors de portée des

30. Il faut ici noter que, pour des raisons évidentes qui tiennent à l'histoire et à l'insularité de la Grande-Bretagne, la mission principale de la British Army a pendant très longtemps été dévalorisée car liée à la police impériale et non à la défense des frontières. Ainsi, s'il y a dans le Royaume une Royal Navy et une Royal Air Force, il n'y a pas de Royal Army mais seulement une British Army. Cela permet dans une certaine mesure d'expliquer encore aujourd'hui le modèle de socialisation professionnelle moins « militarisé » et «conventionnel » de l'armée de terre britannique par rapport à d'autres armées de terre européennes ou, à plus forte raison encore, par rapport à l'armée de terre américaine (sur l'armée américaine voir l'article de Thomas Lindemann dans ce numéro).

31. Voir notamment : colonel Trinquier R., La Guerre moderne, Paris, La Table Ronde, 1960.

32. Le Raj britannique est la dénomination non officielle de la période de domination britannique du sous-continent indien (fin XVIIIe siècle à 1947), c'est-à-dire la zone géographique s'étendant sur les pays suivants : l'nde, le Pakistan, le Sri Lanka, le Bangladesh et la Birmanie.

33. Mockaitis T.R., op. cit., p. 85. 
" populations indigènes », du moins jusqu'à la période des guerres de décolonisation. La rationalité sous-jacente à ces types de pratiques réside entre autres dans une logique de contrôle social devant jouer sur des ressorts interdépendants d'ordre symbolique et matériel, sur des facteurs émotionnels («les cœurs ») et des intérêts (« les esprits »), mais sans que ceux-ci puissent être séparés de logiques purement coercitives. Déjà Lyautey synthétisa cette approche, la dimension fortement idéologisée de la Guerre froide en moins, dans son analyse de la lutte contre « les pirates » en Indochine en 1900 :

«[...] lorsqu'il s'agit de mettre en culture une partie d'un territoire envahi par les herbes sauvages, il ne suffit pas d'arracher celles-ci sous peine de recommencer le lendemain [...]. De même de la terre livrée à la piraterie ; l'occupation armée [...] y passe le soc; l'établissement d'une ceinture militaire l'enclôt et l'isole; enfin la reconstitution de la population [...], l'installation des marchés et des cultures [...] y sèment le bon grain et rendent la région conquise réfractaire au pirate $[. .].]^{34}$ ».

Mais cela n'empêche pas des actions fortement coercitives, comme le montra Lyautey lui-même en 1924, lors de la guerre du Rif au Maroc, à la suite de laquelle la France est accusée d'avoir eu recours à des bombes chimiques contre les villages rifains et lors de laquelle le futur général Beaufre, l'un des théoriciens français de la «guerre révolutionnaire » à la suite la guerre d'Algérie, aura l'occasion de fréquenter Lyautey.

En effet, troisièmement, il faut dissuader la population de coopérer avec l'ennemi en lui montrant qu'il est plus coûteux de coopérer avec celui-ci qu'avec les forces de sécurité. Cela conduit parfois au mimétisme avec le « terrorisme » ou la guérilla comme l'ont montré les militaires américains aux Philippines en utilisant les guérillas supplétives de l'US Armed Forces in the Far East (USAFFE) pour contrer celles du mouvement Hukbalahap (ou «Huk») entre 1945 et 1954. Ce mimétisme par rapport à la stratégie réelle ou perçue de l'ennemi peut alors conduire au recours à la « contre-terreur » ou la « terreur » (mais jamais au «terrorisme ») à travers la guerre psychologique, la torture et la mise en place de structures parallèles calquées sur celles de l'ennemi selon le principe de la contre-organisation (counter-organization). Ces types de pratiques de dissuasion par la «terreur », fondées sur le principe de la punition collective, furent fréquents lors des soulèvements dans les colonies au XIXe siècle sous la forme de massacres, de destructions de villages, du bétail ou des cultures ${ }^{35}$, mais elles perdurent également dans des formes systématisées au XXe siècle notamment lors des guerres d'Algérie et du Vietnam. En jouant sur l'effet psychologique de la guerre et parfois sur la « guerre psycho-

34. Colonel Lyautey, Le Rôle colonial de l'armée, Paris, Armand Colin, 1900, pp 11-12.

35. Colonel Caldwell C.E., Small Wars: Their Principles and Practice, University of Nebraska Press, 1996 [1 $1^{\text {re }}$ publication, 1896]. 
logique » ${ }^{36}$, ces types de pratiques ne peuvent entièrement être distingués de la stratégie « des cœurs et des esprits », si ce n'est qu'il s'agit d'avantage des «esprits » que des « cours » et qu'à la suite de la guerre du Vietnam, il est généralement admis par nombre de militaires que la «punition ciblée » est plus efficace que la « punition collective $» 37$.

Enfin quatrièmement, il faut empêcher l'ennemi d' 'infecter » les populations en s'y « enkystant ». Cette fonction supposerait notamment de contrôler et de surveiller étroitement les populations et le territoire sur lequel ils vivent en le quadrillant. Elle peut aussi prendre l'expression de regroupements forcés de populations ayant vocation à séparer l'ennemi de la population, afin de le priver de tout soutien. Cela peut être fait par des « camps de concentration ", comme cela fut le cas lors de la deuxième guerre des Boers (1899-1902) et l'insurrection philippine de 1899-1902, ou par des villages artificiels fortifiés tels que les "nouveaux villages » mis en place par le plan Briggs en Malaisie, les villages fortifiés installés au Kenya pendant l'insurrection « MauMau » contre l'administration coloniale britannique ou les « hameaux stratégiques » créés en 1961 sous le gouvernement Diem au Sud-Vietnam sous l'influence directe de Sir Robert G. Thompson, un des proches collaborateurs de Harold Briggs, le concepteur britannique des «nouveaux villages » malais, devenu conseiller des forces américaines après la Malayan Emergency.

Bien évidemment, il ne s'agit pas ici de dire que ces différents exemples de «troubles» dans des contextes coloniaux ou post-coloniaux sont identiques ou mêmes similaires. Cependant, ils ont tous conduit les forces expéditionnaires des puissances coloniales à développer des savoir-faire particuliers, cassant la distinction entre ce qui est considéré comme relevant de la sphère interne des Etats - et donc, dans la conception traditionnelle, de la police - d'une part, et ce qui est considéré comme propre à la sphère externe et donc au monde de la diplomatie et de la guerre d'autre part. Ces savoir-faire coloniaux de sécurité et de maintien de l'ordre se développent donc dans les interstices des paradigmes dominants de la sécurité que sont celui de la police en interne et de la guerre interétatique en externe. Ce sont notamment ces savoir-faire centrés sur les populations ("people-centred») qui sont, dans une certaine mesure, importés dans les Opex contemporaines et qui continuent d'en structurer certaines pratiques ${ }^{38}$. On peut ainsi bien s'interroger avec le général Heinrich, ancien commandant en second français des forces de l'ONU (FORPRONU) en Bosnie-Herzégovine au début des années 1990: «la période 60-90 n’a-telle été qu'une parenthèse 39 "?

36. Voir notamment: Géré F., La Guerre psychologique, Paris, Economica, 1996.

37. Maass P., "The counterinsurgent", New York Times Magazine, 11 janvier 2004, p. 32.

38. Bigo D., Terrorisme, guerre, sécurité intérieure et sécurité extérieure, mémoire de HDR en sciences politiques, IEP de Paris, 18 décembre 2002.

39. Général Heinrich J., "Les affaires civiles et le maintien de la paix », IHEDN, n49, avril 1997. 


\section{Modes de transmission et de réactualisation des savoir-faire colo- niaux en Opex}

On peut aborder les continuités coloniales de nombreuses doctrines et pratiques militaires contemporaines - à commencer par les doctrines contreinsurrectionnelles britanniques et américaines, mais aussi la doctrine de l'armée de terre française de la «stabilisation »- de différentes manières. Une première consiste à regarder comparativement les similarités structurelles entre les doctrines contemporaines et les approches de l'époque coloniale. Une seconde manière s'intéresserait d'avantage aux mécanismes sociologiques de transmission et de réactualisation. Nous suivrons ici ces deux voies en gardant à l'esprit que les transmissions historiques au niveau des institutions militaires prises dans leur ensemble sont moins de nature "mécanique » que "réflexive » : elles s'intègrent à des stratégies professionnelles, notamment dans des contextes de remise en question des approches dominantes - tel que la défense territoriale - qui, jusque là, fondaient l'identité militaire.

\section{Les modalités militaires de la " contre-insurrection » en Afghanistan et en Irak}

Avec la fin des guerres de décolonisation - dont la guerre du Vietnam peut être conçue comme un prolongement - les savoir-faire et doctrines contreinsurrectionnelles ont été partiellement marginalisés et relégués à la périphérie du champ de la sécurité. Ainsi, au sortir de la Guerre froide, aux Etats-Unis, seul un petit groupe d'intellectuels de la défense influents, dont Dan Bolger, Eliot Cohen et Steve Metz, défend ouvertement une approche en termes de " contre-insurrection » en dépit du fait que ces doctrines y sont réapparues dès les années 1980 sous la forme des «conflits de basse intensité " ("Low Intensity Conflicts ") ${ }^{40}$. Si les références explicites à ces doctrines réapparaissent sporadiquement avec les Opex des années 1990 - notamment en Bosnie et au Kosovo - ce n'est qu'avec les interventions en Afghanistan et en Irak que ces références deviennent systématiques et officielles.

Pourtant, une fois passée la première phase de l'invasion en Afghanistan en 2001 et en Irak en 2003, l'objectif sera essentiellement pour les troupes américaines, et dans une moindre mesure britanniques, d'éradiquer « les terroristes » par des moyens d'action hautement et quasi exclusivement coercitifs ( «sweep and destroy», "clear and destroy») et en théorie ciblés, s'appuyant sur une croyance aveugle dans le renseignement technologique et les «armes intelligentes ", au besoin complétés par quelques innovations tactiques ${ }^{41}$. Cette approche de la «guerre contre le terrorisme», parfois qualifiée de

40. La parfaite continuité entre contre-insurrection au Vietnam et LIC des années 1980 est mise en évidence dans : Klare M.T., Kornbluh P. (eds), Low Intensity Warfare, Counterinsurgency, proinsurgency and antiterrorism in the eighties, New York, Pantheon Books, 1988.

41. Voir notamment : de Hoop Scheffer A., Hamlet en Irak, Paris, Presses du CNRS, 2007. 
« cinétique 42 » (« kinetic approach»), repose sur l'argument de la puissance de feu écrasante des forces armées américaines. Elle sera surtout promue par la communauté des forces spéciales américaines, réunies au sein du US Special Operations Command (USSOCOM), et par la CIA. Au niveau politique, elle sera relayée par les hérauts de la « force transformation » dont Donald Rumsfeld 43. Cependant, dès le début des interventions en Afghanistan et en Irak, l'influence de la théorie de contre-insurrection se fait progressivement sentir et ce d'autant plus que la stratégie initiale montre ses limites. Cette approche contre-insurrectionnelle devient alors dominante avec la démission de Rumsfeld et la nomination du général David H. Petraeus comme commandant de la «Coalition multinationale Irak » en 2006. En Afghanistan, cette tendance se ressent non seulement dans l'approche de la coalition sous commandement américain (OEF), mais aussi dans la rhétorique de la FIAS, dont nombre de représentants anglosaxons décrivent le mandat comme de la « contre-insurrection », alors que d'autres pays, dont la France et l'Allemagne, s’y opposent, préférant le registre de la « stabilisation ». Pourtant sur ce théâtre, les différences entre ces approches distinctes, ainsi qu'entre la FIAS et l'OEF, sont de moins en moins palpables de l'aveu même de certains acteurs sur le terrain.

L'approche contre-insurrectionnelle en Afghanistan et en Irak, fondée sur le triptyque «clear, bold, build» («nettoyer, tenir, reconstruire») venant compléter des opérations de ratissage plus traditionnelles («cordon and search»), ne fait en réalité que réactualiser d'anciennes recettes coloniales. Il n'est alors pas étonnant qu'un certain nombre de conseillers britanniques aient joué un rôle central en Irak dans l'adoption par l'armée américaine de la rhétorique "des cœurs et des esprits », au risque de froisser les conseillers israéliens qui s'étaient autoproclamés spécialistes de la "gestion des Arabes » en Irak en prônant une approche exclusivement coercitive ${ }^{44}$. L'influence évidente et affirmée des écrits du théoricien français (mais parfaitement anglophone) de la « guerre révolutionnaire », David Galula, sur la nouvelle doctrine américaine de la contre-insurrection atteste également de cette influence coloniale ${ }^{45}$. Dès lors, il s'agit moins ici de mettre en évidence les ressemblances objectives entre les pratiques militaires actuelles en Irak et en Afghanistan d'une part, et celles de la contre-insurrection et de la "guerre révolutionnaire » du temps des guerres de décolonisation d'autre part, que de montrer l'influence indéniable exercée par l'étude de ces dernières sur les premières.

42. L'adjectif «cinétique» (du verbe grec «kinein »: «bouger ») désigne ce qui est relatif au mouvement des corps matériels et des forces qui y sont associées. Exemple : l'énergie cinétique.

43. A propos de la force transformation voir: Wasinski C., "Créer une révolution dans les affaires militaires : mode d'emploi », Cultures $\mathcal{E}$ Conflits, nº4, 2006, pp. 149-164.

44 . Entretien avec un enseignant de la Counterinsurgency Center of Excellence de Camp Tadji, Irak, Grande-Bretagne, 2007.

45. FM 3-24, The U.S. Army/Marine Corps Counterinsurgency Field Manual, University of Chicago Press, 2007 ; Galula D., Counterinsurgency Warfare, theory and practice, Londres, Praeger, 2006 (1 ${ }^{\text {re }}$ édition, 1964), voir notamment la préface de J.A. Nagl. 
Ainsi, l'objectif d'élimination des «terroristes »- essentiellement les sympathisants d'Al-Qaïda, les anciens talibans en Afghanistan et les groupes collaborant avec "l'Etat islamique en Irak » 46 - passe par des techniques et des tactiques qui, non seulement, ne sont pas sans rappeler celles des guerres coloniales mais qui s'en revendiquent souvent ouvertement. C'est notamment le cas du recours de plus en plus systématique à des forces supplétives locales ${ }^{47}$. Celles-ci sont en Irak parfois issues d'anciens groupes « insurgés » comme l'a montré le recours à des membres des « Brigades de la révolution de 1920 » et, dans une moindre mesure, «l'Armée islamique en Irak » (AIS) dans les provinces de Diyala et $\mathrm{Al}$ Anbar en 2007. Par ailleurs, l'incitation à la création et l'armement de comités de salut public antiterroristes tels que l'Al Anbar Salvation Council, les «conseils de l'éveil sunnite» («Sunni Awakening Councils»), les milices des "concerned local citizens» («citoyens locaux inquiets»), ou le fait en Afghanistan d'armer les milices des «seigneurs de guerre ", s'inscrit dans une tradition notamment coloniale, ce dont témoigne le fait que certains de ces contre-guérillas sont parfois comparées aux Macabebe Scouts de la « guerre de pacification » aux Philippines en 1899-1902. Il faut enfin noter que la création d'unités mixtes composées de troupes « internationales» et «locales» en Irak ("Military Transition Teams», MiTT) et en Afghanistan ${ }^{48}$ ne va pas sans rappeler le « jaunissement » et la création du Groupement mixte de commandos aéroportés (GCMA), puis du Groupement mixte d'intervention (GMI) pendant la guerre d'Indochine. Ces dernières sont en effet citées en exemple par les théoriciens américains de la contre-insurrection contemporains 49 .

Concernant la fonction de persuasion, le discours omniprésent sur les « cœurs et les esprits » et les continuités historiques établies par certains militaires américains entre les Provincial Reconstruction Teams (PRTs) - ces équipes civilo-militaires chargées de la protection des populations locales, de l'assistance humanitaire et au développement dans les provinces afghanes et irakiennes d'une part, et le programme CORDS au Vietnam et les SAS en Algérie d'autre part sont révélatrices ${ }^{50}$. Par ailleurs, on notera que l'engagement en 2003 du général Petraeus, alors à la tête de la $101^{\mathrm{e}}$ division parachutiste dans la province de Ninive en Irak, dans l'assistance humanitaire ainsi que la reconstruction éco-

46. On peut cependant noter que, dans certains milieux néo-conservateurs américains, la catégorie de «terroriste» tend potentiellement à s'étendre à l'ensemble des sunnites irakiens. Voir : Hashimi A.S., Insurgency and Counter-Insurgency in Iraq, Ithaca, Cornell University Press, 2006.

47. Pour une tentative de réactualisation des pratiques coloniales de délégation de la pacification à des forces supplétives locales voir : Cassidy R.M., “The long small war: indigenous forces for counterinsurgency”, Parameters, été 2006, pp. 47-62.

48. Operational Mentor and Liaison Teams de la FIAS et Embedded Training Teams de la coalition multinationale sous commandement américain (OEF).

49. Cassidy R.M., op. cit.

50. Entretiens divers réalisés à la Counterinsurgency Academy (COIN Academy) rattachée à Task Force Phoenix/Combined Security Transition Command Afghanistan (CSTC-A), Camp Julien, Darulaman, Afghanistan, septembre 2007. 
nomique et politique ${ }^{51}$ s'inscrit dans cette même perspective de gestion de la population. Elle passe en effet à la fois par l'action sur les variables structurelles et matérielles considérées comme pertinentes à la « gouvernance locale ( «local governance ») des populations et par des actions plus ciblées, plus fines, visant directement leurs représentations et leurs perceptions. L'ensemble de ces activités est ainsi « valorisé » par des « opérations psychologiques » (PSYOPS) suivant une pratique développée à l'époque des guerres de décolonisation. On peut enfin souligner que le débat contemporain entre les tenants de l'approche cinétique et les défenseurs de l'approche contre-insurrectionnelle sur la stratégie à adopter pour éradiquer les « qaëdistes » des régions tribales à la frontière entre le Pakistan et l'Afghanistan est une translation quasi homothétique du débat au sein de l'administration coloniale britannique chargée de la même zone (North Western Frontier) entre la «close-border school » ( l'école de la frontière fermée ») adepte des missions punitives à distance d'une part, et la «forward policy approach » ( "l'approche de la politique d'engagement ») adepte du « hearts and minds» d'autre part au début du $\mathrm{XX}^{\mathrm{e}}$ siècle, soit près d'un siècle plus tôt.

Concernant la fonction de dissuasion par la «terreur » et le contrôle coercitif des populations ${ }^{52}$, le défi est immense en ce que les pratiques résolument illibérales qui en découlent - et l'analyse des généraux putchistes de 1961 est en cela juste - n'est pas compatible avec le cadre formellement libéral des Etats « occidentaux ». Par ailleurs, le déplacement forcé de populations n’est pas conforme au droit humanitaire de la guerre. Ces mesures de contrôle des populations ont alors partiellement été suppléées par la construction de murs autour de certains quartiers sunnites de Bagdad ayant pour but à la fois de séparer les populations des «terroristes » et de les protéger contre les milices chiites. Le quadrillage et l'isolation de villes et de quartiers par le biais de murs ont également été expérimentés dans certaines villes (dont Tal Afar et Falloudjah) ainsi qu'un certain nombre de villages. On peut également évoquer le recours par les Marines à la biométrie (scannage de l'iris) autour de la ville de Falloudjah après la deuxième « bataille de Falloudjah » en 2004, pour empêcher les « terroristes » de rentrer tout en maintenant la liberté de circulation qui n'a jamais été remise en question. Mais cette expérience a constitué un échec patent. Le recours à la torture dans la prison d'Abou Ghraïb et l'existence de centres de détention secrets en Afghanistan - dont il ne semble pas qu'ils puissent être attribués à de simples initiatives individuelles - pourraient en outre laisser entendre que la pratique de la dissuasion par la « terreur » n'est pas forcément inexistante, même si de telles pratiques sont considérées comme contre-productives par la majorité des théoriciens contemporains de la contre-insurrection.

51. Lieutenant-colonel Goya, «Démocratie 101 », Cabiers du CESAT, octobre 2006, pp. 29-35.

52. Pour un tentative de réactualisation des mesures coloniales de contrôle de populations voir : Markel W., "Draining the swamp: the British strategy of population control”, Parameters, printemps 2006, pp. 35-48. 
L'influence des «anciennes» doctrines de la contre-insurrection est aujourd'hui telle que la notion américaine de military nation-building, qui semblait pourtant irrémédiablement décrédibilisée par l'expérience du Vietnam et dont les relents coloniaux étaient évidents malgré le vernis scientifique «fonctionnaliste-systémiste»dont il était paré à l'époque ${ }^{53}$, est aujourd'hui remis à l'ordre du jour. Il importe alors de montrer certain des ressorts par le biais desquels cette réactualisation a pu s'opérer dans un laps de temps qui semble, en surface, avoir été extrêmement court.

\section{Les mécanismes de transmission et de pérennisation des «traditions coloniales »}

On ne saurait en réalité parler de simple « retour » des traditions militaires coloniales pour deux raisons distinctes. La première est d'ordre méthodologique : l'histoire ne se répète jamais à l'identique. Ainsi, lorsque le général Saleun, à la tête du Commandement des opérations spéciales (COS) français lors de l'intervention de la FORPRONU en Bosnie-Herzégovine affirme en 1997 :

« grâce [...] au rayonnement d'un petit groupe d'officiers et de sousofficiers du COS [...], conduits avec clairvoyance par un lieutenantcolonel de la Légion, on a réinventé ces dernières années, les officiers des Affaires indigènes ${ }^{54}$ ",

il ne faudrait pas en tirer des conclusions trop hâtives. D'un point de vue sociologique, cette affirmation en dit bien plus sur le désarroi face aux «nouvelles missions » et sur la volonté de montrer que les Opex renouent avec une tradition ainsi idéalisée, que sur les pratiques matérielles sur le terrain. N'en déplaise à certains «nostalgiques» ou (au contraire) adeptes de la critique facile, le COS n'est pas les Affaires indigènes du temps de Lyautey 55 . Cependant, cela ne signifie pas que les tentatives de réactualisation que révèlent de telles déclarations soient anodines ou sans effets.

La deuxième raison est d'ordre empirique : les lignes de continuité coloniale, si elles ont pu être diffractées dans des directions très différentes et notamment des deux côtés des lignes de démarcation officielle entre « sécurité intérieure » et « sécurité extérieure » dans les contextes post-coloniaux ${ }^{56}$, n’en

53 . Latham M.E., op. cit ; Bilgin P., Morton A., "Historicising representations of 'failed states': beyond the cold-war annexation of social sciences?", Third World Quarterly, vol. 23, n¹, 2002, pp. 55-88.

54 . Général Saleun J., «Actions civilo-militaires : l'expérience en ex-Yougoslavie », in « Des forces spéciales aux affaires civilo-militaires ", Cabiers de la FED, n¹0, 1997.

55 . Il s'agit du service de l'administration coloniale française chargé d'encadrer les populations autochtones sur le plan politique, administratif et social.

56. C'est-à-dire dans un contexte dans lequel « l'entre-deux » colonial, à la fois intérieur (à l'empire) et extérieur (à la métropole) n'est plus officiellement acceptable (à l'exception notable, dans une certaine mesure, de l'Irlande du Nord. 
ont pas pour autant été significativement dénaturées. Ainsi, au travers de leurs avatars successifs dans les interventions extérieures, à aucun moment ces lignes de continuité n'ont été perdues par les acteurs mêmes de cette transmission. Ce sont toujours les mêmes auteurs et les mêmes exemples (notamment la Malaisie) qui sont évoqués malgré les changements de contexte socio-historique. Ainsi il n'y a pas de retour de ces doctrines, dans la mesure où il n'y a pas eu de véritable disparition, mais seulement des périodes successives de marginalisation, de transformation puis de réactualisation.

Les vecteurs de transmission et donc de réactualisation de ces doctrines sont multiples et de différentes natures. On en mentionnera trois ici. Insister sur ces vecteurs est ici d'une importance méthodologique cruciale dans la mesure où cela permet d'insister sur les limites de la lecture fonctionnaliste de ce " retour ». Cela permet en effet de montrer que ce ne sont pas simplement des ressemblances pour ainsi dire objectives entre les conflits contemporains et les « troubles coloniaux » d'antan qui expliquent le retour de ces types d'approches. On n'est pas en la présence d'un simple mécanisme d'adaptation institutionnel impulsé par les réalités matérielles du terrain. Un ensemble de facteurs sociologiques internes aux appareils militaires doit également être convoqué.

Tout d'abord, notamment depuis la guerre du Vietnam, un certain nombre d'opérations militaires seront l'occasion de diffuser plus largement le savoirfaire contre-insurrectionnel et, ainsi, de le revigorer. Parmi celles-ci on peut citer bien sûr les interventions américaines dans les « conflits de basse intensité » des années 1980 (El Salvador, Contras au Nicaragua, etc.) mais aussi et surtout l'intervention des militaires britanniques en Irlande du Nord. En effet, cette dernière sera l'un des biais principaux par lequel certains éléments de la doctrine contre-insurrectionnelle pourront être recyclés puis transnationalisés au travers de l'OTAN dans les «nouvelles » Opex ${ }^{57}$. En effet, même si le terme de contreinsurrection ne sera pas officiellement aposé par les professionnels de la politique à la gestion des « troubles » en Irlande du Nord, il sera fréquemment utilisé par les militaires eux-mêmes et notamment par l'un des concepteurs de l'approche militaire de ces "troubles ", le brigadier Frank Kitson qui, en passant notamment par le Kenya, l'opération de maintien de la paix onusienne à Chypre puis par l'Irlande du Nord, aura appris à jouer des frontières parfois perméables entre différents labels. Il contribuera à diffuser les notions de guerre « de basse intensité », «d'opérations de stabilité » («stability operations ») et même de « maintien de la paix » en tant qu'équivalents sémantiques du concept de contreinsurrection ${ }^{58}$. Ainsi au sein des armées britanniques, le déploiement en Irlande

57 . Voir notamment Bigo D., Guittet E.-P., Smith A., « Participation des militaires à la sécurité intérieure : Royaume-Uni, Irlande du Nord », Cultures E Conflits, n56, 2004, pp. 11-34.

58. A ce propos, il est intéressant de noter qu'un des «pamphlets» officiels sur la «police impériale » et la contre-insurrection britanniques, largement inspiré de l' « Urgence malaise », s'appelait déjà : Keeping the Peace (Duties in Support of the Civil Power), War Office, 1957. 
du Nord sera souvent considéré comme un pré-requis quasiment indispensable pour pouvoir être déployé dans les Opex des années 1990 et notamment dans les Balkans ${ }^{59}$. De ce point de vue, il faut noter qu'en dépit des différences notables entre les doctrines des «opérations de paix » d'une part, celles de la contreinsurrection d'autre part, les premières joueront un rôle important en rassemblant les conditions de possibilité de la « réactualisation contre-insurrectionnelle » actuelle. Ainsi le général David H. Petraeus lui-même n’hésitera pas à affirmer que son passage par la Bosnie aura joué un rôle crucial dans sa prise de conscience de l'importance du type d'approche qu'il adoptera, par la suite, dans la province de Ninive.

Deuxièmement, des échanges transnationaux de doctrines entre la France, la Grande-Bretagne et les Etats-Unis entre autres, pendant toute la période de la Guerre froide, par le biais de conseillers, d'enseignants et d'officiers de liaison, contribueront à pérenniser les «traditions coloniales » tout en les transformant. On verra ainsi par exemple la doctrine française de la « guerre révolutionnaire » influencer significativement l'approche américaine de la contre-insurrection dans les années 1960 à un moment où elle était significativement délégitimée et marginalisée en France ${ }^{60}$. Ces échanges transnationaux sont clairement illustrés aujourd'hui par le véritable «consortium transnational » qui contribua à la rédaction du manuel de contre-insurrection de l'armée américaine et des Marines de $2006{ }^{61}$. En effet, outre Petraeus, y contribuèrent directement le lieutenantcolonel américain John Nagl, vétéran des deux guerres d'Irak et docteur de l'université d'Oxford 62 où il fut largement influencé par la tradition de la contreinsurrection britannique ${ }^{63}$, le colonel américain H.R. McMaster ayant conduit la «pacification» de la ville de Tal Afar en Irak en 2005 et chercheur à l'International Institute for Strategic Studies à Londres au moment de la publication du manuel, le lieutenant colonel australien à la retraite Dr. David Kilcullen, conseiller civil de Petraeus ayant l'expérience de nombreuses missions de contreinsurrection et de maintien de la paix en Asie du Sud-Est, et le colonel britannique à la retraite Gavin Bulloch, spécialiste de la contre-insurrection et ancien Director General of Development and Doctrine au ministère britannique de la Défense. Cette liste est bien évidemment loin d'être exhaustive. Ces militaires ou anciens militaires, influents à la fois dans les milieux des professionnels et des intellectuels de la défense, seront alors les artisans de la réactualisation actuelle et officielle de la «contre-insurrection » à la fois aux Etats-Unis, en Grande-

59. Entretiens divers avec des officiers supérieurs britanniques à Londres entre 2005 et 2007.

60. Voir : Périès G., De l'action militaire à l'action politique. Impulsion, codification et application de la doctrine de la "guerre révolutionnaire " au sein de l'armée française (1944-1960), thèse de doctorat, Paris-I, 1999.

61. Field Manual 3-24.

62. Sa thèse de doctorat, très influente, fut publiée en 2002 (puis rééditée à plusieurs reprises): Nagl J.A., Learning to Eat Soup with a Knife: Counterinsurgency Lessons from Malaya and Vietnam, Chicago, The University of Chicago Press, 2002.

63 . Entretien avec l'auteur, Cumberland Lodge, Windsor, Royaume-Uni, octobre 2007. 
Bretagne, mais aussi dans une certaine mesure au Canada et en Australie. Outre leur provenance des pays du " programme de standardisation ABCA 64 », on notera la bonne connaissance qu'ont ces individus de la tradition impériale et de la contre-insurrection britannique.

Enfin, troisièmement, il faut noter que certains corps militaires seront particulièrement marqués par les traditions militaires expéditionnaires et (néo-) coloniales et viendront jouer un rôle particulier dans la transmission des « traditions militaires coloniales ». Dans le cas américain, on peut notamment mentionner le rôle joué par le US Marine Corps bénéficiant d'une tradition expéditionnaire et d'un savoir-faire hérité notamment des «Banana wars» néo-coloniales en Amérique centrale au début du siècle dernier ${ }^{65}$. Son expérience des guérillas et des «petites guerres » est alors venue lui permettre de faire valoir un savoir-faire et une spécialisation institutionnelle en la matière dans le contexte des interventions en Afghanistan et en Irak ${ }^{66}$.

Pour le cas de la France, c'est notamment le cas des troupes de marine (TDM), « héritières » des troupes coloniales, dont l'histoire vient partiellement se confondre avec celles des guerres coloniales, notamment en Algérie et en Indochine. Or, entre la fin de la guerre d'Algérie et l'engagement de la France dans les « nouvelles Opex » des années 1990, l'essentiel de l'expérience expéditionnaire «non conventionnelle » (à l'exception de la mission au sein de la FINUL au Liban) se retrouvera au sein des TDM, constituant l'essentiel des forces «prépositionnées » en Afrique. Ainsi les TDM, notamment au sein du COS, tenteront au début des années 1990 de jouer un rôle central dans le développement des Opex françaises dans un contexte dans lequel - fait nouveau - tous les corps ont vocation à intervenir sur les théâtres extérieurs. Le savoir-faire colonial sera alors mis en valeur par les TDM dans le cadre de luttes bureaucratiques et institutionnelles autour de la spécialisation dans les Opex. Par ce biais, et dans un contexte où la valeur «stratégique " accordée

64. American, British, Canadian, Australian Armies' Standardization Program incluant la NouvelleZélande promouvant l'interopérationalité en dehors du cadre de l'OTAN. Actuellement, une tentative d'interopérationnalisation des doctrines de la contre-insurrection « anglo-saxonnes » a lieu au travers du programme ABCA à défaut d'avoir réussi à convaincre tous les membres de l'OTAN.

65. Le manuel du corps des Marines sur les Small Wars de 1940, synthétisant les enseignements tirés de ces « guerres de la banane", constitue de ce point de vue un classique. Ce texte remarquable, partiellement réactualisé en 2004, est aujourd'hui considéré par certains Marines comme hautement pertinent pour les opérations en Irak et en Afghanistan. On qualifie d'ailleurs bien souvent ces Marines familièrement de «Banana War Marines"; voir : Small Wars Manual, Washington DC, US Government Printing Office, 1940 :

http://www.au.af.mil/au/awc/awcgate/swm/index.htm

66. Il faut noter que l'identité institutionnelle des US Marines est ambiguë dans la mesure où, historiquement, ils oscillent entre un rôle de force amphibie lourde (sur le modèle de la Seconde Guerre mondiale et de la guerre de Corée) et un rôle de force expéditionnaire spécialisée dans les «petites guerres » sur le modèle des Banana Wars des années 1920 et 1930. Cette dernière tendance sera bien illustrée par le lieutenant général Victor H. Krulak lors de la guerre du Vietnam, puis par la suite par son fils le général Charles C. Krulak. 
aux « opérations de paix » menace de remettre en cause les «valeurs militaires traditionnelles ", ce savoir-faire colonial revendiqué par les TDM, permettra à l'institution militaire de renouer avec des continuités historiques jusque là marginalisées. La valeur de cette spécialisation des TDM est d'ailleurs partiellement reconnue par l'institution militaire : les militaires «projetés » en Opex, quelque soit leurs corps d'appartenance, doivent en général suivre un stage de formation, notamment à la "culture locale ", à l'Ecole militaire de spécialisation de l'Outre-Mer et de l'étranger (EMSOME) liée aux TDM. Par ailleurs, la reconnaissance internationale n'est pas en reste: lorsque les US Marines créeront un Cultural Centre of Excellence au sein de leur base de Quantico en Virginie - la «sensibilité culturelle» («cultural awareness») et le recours à des « anthropologues culturels » étant considérés comme indispensables à la stratégie « des cœurs et des esprits »-ce centre coopérera notamment avec les French Marines, c'est-à-dire les TDM.

On observe donc un ensemble hétérogène de mécanismes qui ont pour mérite de souligner qu'on n'est pas en la présence d'une simple adaptation fonctionnelle aux singularités de l'environnement « opérationnel » en Afghanistan et en Irak. Si adaptation il y a, celle-ci mobilise également des mécanismes bien connus de l'analyse décisionnelle et qui consistent en des processus cognitifs au travers desquels les acteurs tendent à intégrer des informations nouvelles dans des représentations déjà établies et inscrites dans une histoire longue ${ }^{67}$. Mais les éléments susmentionnés permettent également de montrer que ces mécanismes cognitifs n'opèrent pas dans un « vide » social. Ils sont au contraire sous-tendus par des ressorts - des réseaux sociaux, des luttes bureaucratiques et des « réinventions de la tradition »- objectivables par l'analyse sociologique.

Les différents éléments abordés jusqu'ici nous semblent poser un ensemble de questions plus générales. Dès lors, il nous reste désormais à expliciter les enjeux théoriques et pratiques que les réactualisations coloniales soulèvent concernant les conditions de possibilité politiques de l'actualisation de leur objectif formel de «pacification », de «stabilisation » ou de «normalisation » en Opex.

\section{De la mort des empires aux Opex : les paradoxes modernes de la légitimation du recours à la force}

Il faut ici commencer par dissiper un doute. L'« empire global », et a fortiori "l'empire global américain », n'existe pas. Qui plus est, contrairement à ce qu'affirme une certaine idée en vogue au sein de l'ultra-gauche, nous n'assistons pas au retour des empires d'antan. Tout au plus y aurait-il des tentations impériales.

67. Jervis R., Perception and Misperception in International Politics, Princeton, New Jersey, Princeton University Press, 1976. Pour une analyse de l'isomorphisme relatif aux " adaptations » des institutions militaires voir : Farrell T., Terriff T., The Sources of Military Change: Culture, Politics, Technology, Boulder, Lynne Rienner, 2001. 
Mais celles-ci viennent aujourd'hui inévitablement se briser, telles des vagues, sur les sables de la modernité politique. Cette dernière tient en effet l'empire en horreur ${ }^{68}$. Mais alors, pourquoi insister sur les antécédents coloniaux de certaines des pratiques militaires contemporaines ? Est-ce que le fait qu'une pratique prenne ses sources dans une période antérieure démontre qu'elle n’a pas changé depuis ? Est-ce que cela permet de transposer des enseignements politiques antérieurs dans le présent ? Assurément non. Dès lors, l'intérêt de la démarche historique est ici de «visibiliser » un certain nombre d'éléments que la simple analyse du temps présent ne permet bien souvent pas de voir. Elle permet notamment de montrer « l'affinité élective » existant entre contre-insurrection d'une part et des techniques spécifiques de contrôle de populations, dont il faut ici analyser les caractéristiques, les enjeux et les problèmes, d'autre part.

\section{L'enjeu de la légitimation "locale » de la force en Opex}

Les traditions doctrinales mobilisées en Afghanistan et en Irak prescrivent une combinaison de mesures d'encadrement, de quadrillage, de coercition, de protection, d'incitation, de dissuasion et de « séduction » par des opérations et des actions psychologiques et « civiques » («civic action»), comme remède à la violence politique. Ces mesures ont toutes en commun de placer la catégorie de « population » au centre des considérations stratégiques. Il s'agit de la « population » comme tiers arbitre (et donc comme « public»), comme cible de la stratégie de persuasion, mais aussi comme milieu d'évolution de «l'ennemi ». Une multiplicité de «tactiques » est alors mise en place qui considère cette population à la fois objectivement du point de vue de sa relation à son milieu et subjectivement du point de vue de ses perceptions. Il s'agit en effet de mener une " guerre totale 69 » selon l'expression de John D. Waghelstein, colonel à la retraite des forces spéciales américaines qui commandait dans les années 1980 le US Military Advisory Group à El Salvador et qui enseigne désormais la contre-insurrection :

« la guerre totale veut dire que l'on utilise toutes les composantes de la puissance nationale [...]. On ne peut se restreindre à n'utiliser que les militaires. Il faut qu'il y ait un flux constant de propagande ; il faut qu'il y ait du soutien économique; il faut qu'il y ait des élections. Aussi longtemps que l'on se contente de chasser ceux qui portent des armes, on néglige l'aspect le plus important 70 ».

68. Walker R.B.J., «L'international, l’impérial, l'exceptionnel », Cultures E Conflits, n`58, 2005, pp. 13-52.

69. L'acception de l'expression est cependant légèrement différente de celle qui lui est généralement attribuée, notamment à la suite du général Beaufre. Cependant l'acception de Waghelstein, caractéristique des conflits de basse intensité des années 1980, est aujourd'hui reprise par certains des théoriciens contemporains de la contre-insurrection en Afghanistan et en Irak et notamment par le lieutenant-colonel John Nagl.

70. J. Waghelstein, cité dans Maass P., “The counterinsurgent”, op. cit., p. 35 (notre traduction). 
Plus que d'une tactique ou d'un mode d'action particulier, le leitmotiv « des cœurs et des esprits » devient alors une stratégie générale.

$\mathrm{La}$ "pacification », ce troisième terme qui vient s'introduire entre la guerre et la paix, se distingue ainsi bien de la simple soumission, du pouvoir d'assujettissement, par la punition collective. Afin d'éclairer cette distinction, l'analyse foucaldienne est particulièrement heuristique d'une part parce qu'elle empêche de considérer le " pouvoir » comme un phénomène unitaire et univoque en invitant à saisir ses éléments d'hétérogénéité les plus subtils, d'autre part parce qu'elle insiste sur l'émergence de la notion de " population » en tant que catégorie de gouvernement au XVIII siècle européen ${ }^{71}$. Enfin, elle montre que le mode contemporain de gouvernement des populations - celui de la gouvernementalité qui s'exerce au nom de leur sécurité à l'instar des Opex contemporaines - n'a pas émergé ex nibilo du «pouvoir souverain» de tuer, caractéristique de l'époque féodale. La transition, inscrite sur plusieurs siècles, s'est notamment faite via le «moment disciplinaire » autour des XVIe et XVII e siècles où le pouvoir vient progressivement à s'exercer de manière plus « efficace », « économique » et fine, notamment par la mise en place de mécanismes de surveillance des individus et de quadrillage du territoire ${ }^{72}$.

Or, il faut de ce point de vue noter que les discours et pratiques militaires dans les contextes coloniaux et dans les Opex contemporaines reprennent précisément les catégories caractéristiques du «moment disciplinaire » décrit par Foucault. Il en est ainsi de la notion de «quadrillage » que l'on retrouve dans la pensée militaire française dans les années 1960, entre autres dans les travaux du général Gambiez sur le "quadrillage de souveraineté 73 ». Elle persiste aujourd'hui en tant que mode d'action central à la « pacification » des populations en externe. Par ailleurs, historiquement le pouvoir disciplinaire s'exerce de manière plus rationnelle et économique que le pouvoir souverain de tuer : les moyens utilisés sont moins visibles et cruels, et donc moins susceptibles d'attiser l'insoumission et les résistances populaires, alors que ses effets sont démultipliés ${ }^{74}$. Or, cet élément central se retrouve aussi dans le discours militaire sur les Opex : le recours à la force massive est contre-productif et

« pendant la stabilisation du thêâtre, un emploi limité et pertinent de la force conduit plus sûrement au succès que ne le ferait son usage immodéré. Veillant à ne pas nourrir une violence qu'elle cherche à maitriser, une troupe doit chercher à imposer sa force, si possible en ne l'utilisant pas ${ }^{75}$ ».

71. Foucault M., Sécurité, territoire, population, Cours an Collège de France, 1977-1978, op. cit.

72 . En réalité, et Foucault revient à plusieurs reprises sur ce point, il ne s'agit pas tant de phases successives que d'un processus cumulatif : le pouvoir souverain demeure au sein du pouvoir disciplinaire et de la gouvernementalité.

73. Gambiez F., Suire M., L'Epée de Damoclès, la guerre en style indirect, Paris, Plon,1967.

74. Foucault M., Surveiller et punir, Naissance de la prison, op. cit.

75 . CDEF, «Gagner la bataille, conduire à la paix : les forces terrestres dans les conflits aujourd'hui 
Enfin, le mouvement disciplinaire et plus encore celui de la gouvernementalité est aussi celui à partir duquel les modalités d'exercice du pouvoir se diversifient considérablement au-delà de la coercition physique. Se mettent en place des dispositifs fondés sur la prise en charge administrative, économique, sanitaire et psychologique des individus et des populations. On retrouve là le phénomène de diversification des répertoires d'action militaires en Opex à travers une démultiplication des « lignes d'opération logiques » (économique, politique, sanitaire, humanitaire, psychologique) devant concourir à l'objectif politico-stratégique.

Dès lors, les doctrines contre-insurrectionnelles révèlent-elles une logique de disciplinarisation des individus par le biais de la surveillance et du quadrillage ou faut-il les analyser en termes de gouvernementalité fondée sur la gestion de la population ? En réalité, Foucault rappelle que les mécanismes de la discipline n'ont jamais été aussi importants que lorsqu'on a essayé de gérer les populations. Ainsi, si la corrélation principale que révèlent ces doctrines est certainement celle entre technologies de sécurité d'une part et population d'autre part, elles ne s'en inscrivent pas moins dans une logique disciplinaire. De la même façon, elles n'excluent pas le pouvoir souverain de tuer, loin s'en faut. Il est important d'insister sur ces éléments parce qu'ils montrent que les principes (mais non les pratiques) sous-jacents à ces approches militaires «population-centrées » ne se distinguent guère significativement de ceux des modalités plus générales d'exercice du pouvoir politique contemporain - si ce n'est que l'environnement conflictuel dans lequel ils sont mis en œuvre leur confère certainement une dimension paroxystique particulière.

Mais ils s'en distinguent de par leur contexte spatio-temporel spécifique. Le problème de la population en tant qu'objet de technologies de pouvoir, historiquement, a en effet été celui de la ville, celui de la ville franche de l'époque féodale qu'il a fallu progressivement réconcilier avec la légitimé de la souveraineté territoriale. Il n'est alors pas étonnant que la ville occupe une place particulière et privilégiée dans de nombreuses théories de la contre-insurrection ou de la guerre révolutionnaire ${ }^{76}$. Mais quels sont alors, en Opex, ces deux éléments qu'il faut savoir réconcilier ? Qui est souverain légitime dans le contexte des «conflits de projection » ? En d'autres termes, si l'enjeu sousjacent à la « conquête des cœurs et des esprits » est celle de la légitimation locale du recours à la force militaire 77 comme tendent à le montrer les références au local consensus building, à la mission acceptance ${ }^{78}$, à l' " adhésion ", $\mathrm{au}$ « consentement », à « l'approbation » et parfois - de façon plus réaliste il

et demain ", FT01, janvier 2007 p. 44.

76. Voir notamment : Hoffman B., Taw J., The Urbanization of Insurgency: The Potential Challenge to US Army Operations, Santa Moncia, Rand Corporation, 1994.

77 . Nous employons ici le concept de légitimation au sens de Lagroye : Lagroye J., « La légitimation », in Grawitz, M., Leca, J., Traité de sciences politiques, t. 1, Paris, PUF, 1985, pp. 395-467.

78. Ces deux expressions couramment utilisées dans le vocabulaire militaire anglo-saxon désignent respectivement la « construction d'un consensus local » et « l'acceptation (locale) de la mission ». 
faut le dire - à «l'acquiescement» de la population, qui cette légitimation doit-elle servir ? Officiellement, il ne fait pas de doute qu'il s'agit des « gouvernements locaux ». Mais, comme l'affirme le manuel américain de la contreinsurrection, des "efforts en vue de construire un gouvernement légitime par des moyens illégitimes sont contre-productifs 79 ». En d'autres termes, l'enjeu de la légitimation ne concerne pas uniquement les gouvernements locaux mais également les forces militaires « étrangères ».

\section{La modernité politique contre la légitimation « externe » de la coercition physique}

A ce stade de l'analyse, l'affirmation selon laquelle les principes (mais non les modalités concrètes) des approches contre-insurrectionnelles ne se distinguent que peu des dispositifs de pouvoir contemporains à l'intérieur des Etats "occidentaux », ne saurait surprendre. Il a en effet déjà été souligné que la contre-insurrection "moderne », en externe, s'apparente dans une certaine mesure à des missions de sécurité intérieure par des moyens militaires dans des Etats tiers. Ainsi elle casse la distinction entre défense et sécurité intérieure. D'ailleurs, dans le vocabulaire militaire anglo-saxon, l'approche contre-insurectionnelle est parfois décrite par le concept de «foreign internal defense » (FID) qui, en insistant sur la nécessité de déléguer la mission de défense aux « forces de sécurité locales », illustre bien le brouillage entre défense extérieure et sécurité intérieure. En ce sens, il n'est pas étonnant que des traditions doctrinales coloniales aient pu être mobilisées et paraître faire sens. Ces traditions sont en effet nées d'un contexte historique où, précisément, des territoires «allogènes " étaient intégrés politiquement à l'intérieur d'un empire colonial et cela, même si ce processus s'accompagnait généralement d'un processus contradictoire d'aliénation politique de populations ainsi indigénisées.

Or, nous sommes là en face d'un paradoxe : contrairement au processus de formation de l'Etat contemporain «en interne » et, dans une moindre mesure, à la «police impériale»dans les contextes coloniaux, ces missions ne peuvent aujourd'hui se présenter autrement que comme des «opération extérieures». Les frontières entre l'interne et l'externe sont en effet déjà tracées - bien qu'elles soient de plus en plus mouvantes 80 - et prédéterminent ainsi les conditions de la légitimation de la force. Réconcilier la légitimité de la souveraineté et le fait de la ville (la population), corréler la légitimation des technologies de sécurité et la population « en interne » a été dans les Etats européens un processus relativement progressif et sans soubresauts majeurs. En effet, il n'y avait que peu de « relations à distance 81 » préexistantes, frontaliérisées et d'ordre politique susceptibles de remettre en question et de concurrencer les relations symboliques et matérielles (notamment fon-

79. FM 3-24: I-132.

80. Walker R.B.J., Inside/Outside, International Relations as Political Theory, Cambridge, Cambridge Studies in International Relations, 1993.

81. Nous empruntons ici cette notion à Gérard Noiriel, op. cit. 
dées sur la citoyenneté et la nationalité) établies par l'Etat moderne en gestation. Il n'y avait pas de grand narratif politique alternatif qui ait été intériorisé par « les populations » au point de pouvoir prédéterminer les conditions de la légitimation de la coercition physique à l'échelle de l'Etat territorial.

Mais précisément, les contextes socio-historiques des Opex contemporaines ne sont pas pré-modernes. Elles sont structurées par des « relations à distance » d'appartenance et d'indentification - même si elles ne sont pas nécessairement nationales ou territorialisées - par rapport auxquelles les « forces intervenantes » en Opex sont par définition extérieures. La distinction entre l'interne et l'externe y fait donc suffisamment sens pour que les « forces internationales » puissent être perçues comme violant les normes qu'elles ont précisément comme mandat de défendre sur le terrain, c'est-à-dire celles de la « souveraineté locale » (présupposant un interne et un externe) qui définissent et constituent l'autorité politique à l'ère de la modernité politique. La stratégie de nation building militaire - qui dit d'ailleurs de plus en plus son nom en Afghanistan et en Irak - s'apparente alors effectivement à un conflit de légitimité dans lequel les forces intervenantes souffrent d'un handicap structurel de poids, celui d'être précisément des «forces intervenantes » et donc extérieures. Dès lors, comment des principes qui ont généralement réussi à s'imposer à l'intérieur des Etats contemporains au terme d'un processus s'étalant sur plusieurs siècles, mais qui n'ont pas atteint leur objectif dans les contextes socio-historiques coloniaux, pourraient-elles désormais réussir dans le cadre d'Opex et ce, qui plus est, dans un laps de temps relativement court ? Le réponse est certainement à trouver dans l'option du «transfert des fonctions de sécurité » aux autorités locales après le transfert formel de souveraineté. Mais la difficulté théorique et pratique est de savoir comment distinguer un tel transfert d'une simple stratégie de délégation - et rendre cette distinction crédible - étant entendu que la souveraineté est par définition, en principe du moins, ce qui ne se transfère pas.

Comme le note alors très justement le général Rupert Smith dans son langage propre :

« chercher à capter la volonté d'une population tout en combattant l'adversaire en son sein est souvent très difficile à réaliser dans des circonstances dans lesquelles les deux - adversaire et population appartiennent à une seule nation ou ethnicité face à une force intervenante. La confrontation sous-jacente est trop facilement réduite par l'adversaire à une opposition entre "eux et nous" et ainsi diffusée au travers de la zone conflictuelle 82 ».

82. Smith R., The Utility of Force, The Art of War in the Modern World, Londres, Allan Lane, 2005, p. 395. 
Dire cela ne revient pas à avoir une conception fixiste des frontières. Les frontières entre l'interne et l'externe sont, de fait, mouvantes. Mais cela joue aujourd'hui en Afghanistan et en Irak en la défaveur de l'objectif officiel de "pacification ». En effet, il conduit à reproduire ces frontières au sein même des sociétés qu'on entend voir revenir à la " paix civile » : pour les autorités locales, l'ennemi public - traditionnellement considéré comme ennemi extérieur devient un ennemi intérieur qu'il faut combattre à tout prix, alors même que, pour ses opposants, les représentants gouvernementaux peuvent désormais être décrits comme alliés à « une force d'occupation ». Cela est d'autant plus le cas que les approches contre-insurrectionnelles de la «population locale » ont souvent, pour des raison historiques, des affinités évidentes avec des lectures ethnographiques dans lesquelles la focale «tribale ", "clanique» ou " ethnique» prend une importance telle qu'elles ne manquent pas d'induire des prophéties auto-réalisatrices sur le terrain. Il n'est alors peut-être pas étonnant que la stratégie contre-insurrectionnelle en Irak ait fini par actualiser un des seuls principes que les puissances coloniales se sont toujours refusées à mettre en œuvre au sein de la métropole : celui du divide ut imperes, diviser pour régner, consistant, en Irak, à armer des groupes et des milices opposées, dans l'espoir ultime que si on ne peut y exporter la "paix civile», au moins, peut-être, pourra-t-on y importer le principe de l'équilibre de la puissance censé régir les relations internationales, entre Etats différents voir opposés...

La pérennisation des présences militaires dans les "guerres» en Afghanistan et en d'Irak - parfois rassemblées dans le discours stratégique américain au travers de la notion de "guerre longue 83 » suscite de nombreux dilemmes. En Afghanistan, on envisage de plus en plus que la «guerre » - à l'instar des guerres de décolonisation - dure encore vingt ans ${ }^{84}$. Pourtant, nombreux sont les militaires à insister sur le dilemme apparemment insoluble auquel ils font face : leur présence participe de la dynamique de radicalisation $\mathrm{du}$ conflit, mais celui-ci aurait pris des proportions telles qu'un retrait rapide ne permettrait pas de maintenir un rapport de force favorable au gouvernement en place. La force viendrait pallier le déficit de légitimité mais, ce faisant, se substituerait à elle...

Pourtant, si dans ce contexte, la «conquête des cœurs et des esprits» apparaît comme un mécanisme correcteur des effets délétères d'une présence prolongée, elle constitue autant le nœud même du problème. Bien sûr, on ne peut qu'encourager les appels répétés à la « sensibilité culturelle » et à l'arrêt des «frappes indiscriminées » ou «mal ciblées » ${ }^{85}$, mais lorsque ces mesures se mettent en système au travers d'un discours mobilisant des savoir-faire coloniaux de gestion de population, le phénomène de la pérennisation de la

83. US DOD, Quadrennial Defense Review (QDR), février 2006.

84. Entretien au COIN Academy, Camp Julien, Darulaman, Afghanistan, septembre 2007.

85 . Voir dans ce numéro l'article de Thomas Lindemann. 
présence militaire « internationale » devient inévitable. Ainsi, alors même que leur objectif est la légitimation «locale»du recours à la force, ces mesures semblent autant susceptibles de crédibiliser les perceptions des « forces internationales » comme forces d'occupation. De ce point de vue - et cela alors même que nombre de ses adeptes insistent sur la «nature politique » de toute insurrection - la stratégie de la contre-insurrection apparait de plus en plus comme un substitut à une stratégie de sortie politique du conflit... 